Cases are children $<16$ years who attend any paediatric trauma hospital in the state of New South Wales, Australia. Controls are age and gender matched children who have ridden off-road vehicles in the last year, recruited through social media and advertising through riding and training organisations. Case numbers will be limited by the number of children presenting to hospital, we expect a final sample of 50-70 children. Our aim is to collect 2-3 controls per case, so the estimated control sample size is 210. For cases, data collection involved in-depth interviews, inspection of the crash site, vehicle and protective equipment. For controls, data collection is via an online survey. Information on riding history, training, behaviours, locations and purpose, as well as rider anthropometry, vehicle type and protective equipment will be collected from both samples. Conditional logistic regression modelling will be used to conduct the case-control analysis.

Results To date, 20 cases have been recruited and 40 control riders. Of the first 12 cases with complete data, all have been males aged between 4 and 15 years. Almost all (11/12) wore helmets and full protective gear at the time of the crash. Injury severity ranged from AIS1-5. Most crashes involved a loss of control or impacting fences.

Conclusions This study will provide an in-depth analysis of risk factors related to children riding powered off road vehicles. It could inform the development of injury preventative strategies in terms of regulation, training, legislation, licensing, vehicle and equipment design and manufacture.

\section{THE BIRTHDAY PARTY}

Christie Burnett, Elizabeth Lumsden. Community Safety Project Officer, RSPA, Community Safety Manger, RSPA

\subsection{6/injuryprev-2016-042156.657}

Background The importance of teaching home safety to children in Scotland has been highlighted by NHS Scotland statistics, which show 2,128 injuries to children under five happen in the home each year. Most of these accidents are preventable through improved awareness, improvements in the home environment and greater product safety.

Methods RoSPA worked in partnership with Go Safe Scotland, an online education resource, and author Linda Strachan, to produce 'The Birthday Party', a book aimed at children 6 years old and younger. The book was produced to highlight home safety issues in the form of a fun and engaging story. Funds were raised through various sources to be able to produce a printed copy of 'The Birthday Party' and allow it to be distributed to Primary One pupils in Scottish schools.

Results RoSPA have still to collect feedback on the resource from primary schools however the production of 'The Birthday Party' has engaged the attention of a wider audience and has appealed to those who work with children and families. Requests have come in from a number of organisations including: The Royal Hospital for Sick Children in Edinburgh, Community Safety Partnerships and Police Scotland. The production of the book has allowed RoSPA to make new partnerships with organisations they have never worked with before, helping to reach the desired target audience and risk groups of vulnerable families across the whole of Scotland.

Conclusions 'The Birthday Party' has been well received throughout Scotland by leading health and safety organisations and Scottish schools. The distribution of the book has allowed us to target our most vulnerable age groups. By implementing home safety issues and preventative measures in a fun and engaging way we have been able to introduce new desirable behaviour for young children. Through producing the book along with Go Safe Scotland we have been able to co-ordinate teaching notes to allow follow up sessions after reading the book with mum/dad/ carer to enhance the learning and increase awareness around home safety.

\section{SMART SHARE OF SERIOUS INJURY AMONG SCHOOLS IN THE ERA OF CLOUD COMPUTING}

1,2 Kenta Imai, ${ }^{1,3}$ Koji Kitamura, ${ }^{1,3}$ Yoshifumi Nishida, ${ }^{2}$ Hiroshi Takemura, 1,3,4 Tatsuhiro Yamanaka. 'National Institute of Advanced Industrial Science and Technology, Japan; ${ }^{2}$ Tokyo University of Science, Japan; ${ }^{3}$ Safe Kids Japan, Japan; ${ }^{4}$ Ryokuen Children Clinic, Japan

\subsection{6/injuryprev-2016-042156.658}

Background A large number of injuries occur in Japanese school environments. To prevent injuries in school, it is important to share injury data and find serious injuries that we have to deal with. In this paper, we developed a cloud computing system for sharing serious injury among schools.

Methods We develop the following method; First, using text mining, we obtain feature values expressing characteristics of the situation for each situation data. Second, we detect serious injury situation that should be shared among schools by evaluating both similarity among environmental characteristics of schools and medical cost. We use 5,817 injuries from sixty nine Japanese schools' injury data to evaluate the developed system in cooperation with Japan Sport Council. This injury data include Japanese elementary and junior high school injury data.

Results The developed system classified 5,817 injury data were into twenty three groups and could detect serious injuries for each group. The developed system also enables us to recommend serious situations that a school risk manager should know. For example, when a user inputs "a student was playing tag and hit his head on a chin-up bar." into the system, the system outputs "a student was playing with ball after school. He ran after the ball and hit his nose on a chin-up bar." The output injury case is similar to the input injury case but it is much more serious since bone fractures occurred. Thus, system allows us to share serious injury situation among schools.

Conclusions We describes a new system for sharing serious injury in a new manner that fits with the era of cloud computing. Disseminating the developed software to more schools and creating community for school safety is an important future work.

\section{THE ROLE OF BODY MASS INDEX ON CHILDHOOD PEDESTRIAN INJURY RISK}

${ }^{1}$ Elizabeth E O'Neal, ${ }^{1}$ Plumert M Jodie, ${ }^{2}$ Leslie A McClure, ${ }^{3}$ David C Schwebel. ${ }^{1}$ University of lowa, USA; ${ }^{2}$ Drexel University, USA; ${ }^{3}$ University of Alabama at Birmingham, USA

\subsection{6/injuryprev-2016-042156.659}

Background Road traffic deaths rank as the $2^{\text {nd }}$ leading cause of death worldwide in children aged 5 to 9 years old. Child pedestrians are particularly vulnerable. Many risk factors have been identified as contributing to childhood pedestrian injury risk, but the role of obesity is less well understood. Obesity affects children's physical and cognitive abilities, both of which could 